\title{
A QM/MM study of acylphosphatase reveals the nucleophilic- attack and ensuing carbonyl-assisted catalytic mechanisms
}

\author{
Zheng Zhao ${ }^{1,2^{*}}$, Philip E. Bourne ${ }^{1,2}$, Hao $\mathrm{Hu}^{3}$, and Huanyu $\mathrm{Chu}^{4}$
}

1. School of Data Science, University of Virginia, Charlottesville, Virginia 22904, United States of America

2. Department of Biomedical Engineering, University of Virginia, Charlottesville, Virginia 22904, United States of America

3. Department of Chemistry, Hongkong University, Hongkong

4. School of Life Science, University of Science and Technology of China, China

*Corresponding author

Zheng Zhao: zz7r@virginia.edu 


\begin{abstract}
Acylphosphatase (ACP) is one of the vital enzymes in many organs/tissues to catalyze an acylphosphate molecule into carboxylate and phosphate. However, the specific catalytic mechanism still remains debated. Here we use a combined ab initio quantum mechanism/molecular mechanism (QM/MM) approach to reveal the catalytic mechanism. We applied a multi-dimensional reaction-coordinates-driving scheme to the benzoylphosphate-bound acylphosphatase system. We obtained a detailed catalytic process including one nucleophilic-attack and then an ensuing carbonyl-shuttle catalytic mechanism by calculating two-dimensional potential energy surfaces. At the same time, we obtained an experiment-agreeable energy barrier and validated the role of the key amino acid Asn38. Additionally, we also qualified the transition state stabilization strategy based on the amino acids-contributed interaction networks revealed in the enzymatic environment. This study also provided multi-scale reaction insights into underlying catalytic mechanism of acylphosphatase to contribute to disease-involved research, such as for early-onset familial Alzhemer's disease.
\end{abstract}




\section{Introduction}

Acylphosphatase (ACP, E.C. 3.6.1.7), which generally consists of $\sim 100$ residues, is one of the smallest enzymes. However, it efficiently catalyzes the hydrolysis of the carboxyl-phosphate bond of the acylphosphate molecule ${ }^{1-2}$ ACP is widely distributed in different organs and tissues, such as muscles and the liver ${ }^{3}$, and plays very important role of metabolism and physiology as an intermediate in the tricarboxylic acid cycle, glycolysis, urea biosynthesis and pyrimidine and in the activity of membrane ion pumps ${ }^{4-7}$. Recently, in cultured skin fibroblasts of patients, ACP has been found to be affected by early-onset familial Alzhemer's disease ${ }^{8-9}$. Despite the increasing number of verified physiological and metabolic processes that involve $\mathrm{ACP}^{10-11}$, the intrinsic catalytic mechanism of acylphosphatase is still being debated.

To reveal the catalytic process, multiple researchers studied the catalytic mechanism of $\mathrm{ACP}^{12-16}$. Saudek and Taddei initially discovered the active site located near the C terminal $^{12,15}$. Taddei's research further revealed the residues Arg20 and Asn38 close to the active site (also marked Arg23 and Asn41 in some PDB structures ${ }^{4}$ ) are very essential to the catalytic performance of mammalian $\mathrm{ACP}^{15-16}$. In another paper, Satchell found that one crystal water molecule at the active site was used as the nucleophile in the process of catalysis $^{13}$. The crystal water-involved catalytic process was also roughly tested in our paper by a two-step process as shown in Figure 1(Scheme 1): the first step is the hydrolysis of the water molecule by attacking one hydrophilic amino acid, and then the second step is the breaking of the carboxyl-phosphate bond ${ }^{13}$. Currently, multiple ACP structures from different organisms have been released and provided the structural basis for exploring the catalytic mechanism ${ }^{17-23}$. Thunnissen et al. determined an ACP structure by using the X- 
ray crystallography method from bovine testis. Based on this, they suggested a similar reaction scheme to Scheme 1 (Figure 1), in which the water molecule was thought to form a hydrogen bond with Asn38, and the phosphate moiety first gains a proton from the water molecule. Then the transition state is formed by the attack of the hydroxyl group ${ }^{21}$. Moreover in 2005, Cheung et al. crystalized one ACP structure from the hyperthermophilic archaeon: Pyrococcus horikoshii $(\mathrm{PhAcP})^{5}$. In the crystal structure, one formate ion and three water molecules were found at the active site. By considering the acetyl-phosphatase similar to the formate molecule and the water molecules, the author infers that the Asn38 residue plays an important role in binding the nucleophilic water molecule at the active site and then forming a hydrogen bond with the non-bridging oxygen atom of the phosphate group. Additionally, the Arg20 residue forms a hydrogen bond to the carbonyl group of the substrate, and the Val14 residue forms a hydrogen bond to the crystal water molecule. The reaction steps are similar to Scheme 1 by following a two-step procedure: firstly, the proton was transferred from crystal water to an oxygen atom of the phosphate group; then the translation state was formed in the concerted process of hydroxyl anion attacking the phosphorus atom and the leaving group decomposing. In 1997, Stefani et al. provided another kind of reaction scheme based on their released X-ray structure as shown in Figure 1(Scheme 2). A proton from the crystal water molecule first formed a transition state between the oxygen atom of a water molecule, the carbonyl oxygen atom of Asn38 and an oxygen atom of the phosphate moiety. Then the proton and the oxygen atom of the water molecule concertedly attack the oxygen atom and phosphorus atom of the phosphate moiety respectively ${ }^{4}$. Although these structure-based insights show different catalytic pictures $^{21,24}$, they hint at possible catalytic details. Following this, we can propose another 
scheme as shown in Figure 1 (Scheme 3). In this scheme, the first step is the oxygen atom of a water molecule as a nucleophile directly attacks the phosphate group, and then the proton of the water molecule is transferred to the oxygen atom of the phosphate group. Of course, in light of new enzymatic applications and disease-involved significance of ACP, we hope to reveal a clear catalytic mechanism, and the specific functional role of the amino acids constituting the catalytic environment.

In this paper, we focus on addressing the catalytic mechanism and calculating the catalytic protocols in detail by exploring multi-dimensional potential energy surfaces using $a b$ initio $\mathrm{QM} / \mathrm{MM}$ energy functions. Firstly, we note that ACP does not released any 3D Michaelis-Menten complex or Michaelis-Menten transition state conformation. We obtained the initial Michaelis-Menten complex structure using on-line web-servers and docking tools ${ }^{25-26}$. Then we explore all possible reaction schemes (Scheme 1-3) with a multi-dimensional reaction-coordinate driving method without any restraint. Additionally, to check how Asn38 partakes in the catalytic process, we set up two sets of different reaction coordinates (including Asn38 or not) for Scheme 1-3 to compare. Finally, we reveal a preferred nucleophilic-attack and ensuing carbonyl-assisted shuttle catalytic mechanism as well as the reaction characters in agreement with the experimental data.

\section{Materials and Methods}

\section{Reaction systems preparation}

We used one X-ray structure of ACP from Pyrococcus horikoshii ( $\mathrm{PhAcP})$ complexed with formate (PDB id: 1w2i) to prepare the enzyme system ${ }^{5}$, and used one benzoylphosphate molecule as the substrate (i.e. $\mathrm{R}=$ benzoyl in the paper in Figure 1 or $3 \mathrm{a}$ ). The 
benzoyl-phosphate molecule was built by Corina online demo. ${ }^{25}$ To obtain the enzymesubstrate complex, we docked the benzoyl-phosphate molecule into the binding pocket using Autodock 4.0 packaged with AutoDockTool1.5.6 $6^{26}$. In the process of docking, the enzyme system, including all protein atoms and one crystal-water molecule (A2046) in the active site, was first added to the polar hydrogen. Then a macromolecule-center grip map of $56 \times 56 \times 56$ grid points and 0.375 spacing was generated by using the Autogrid program. The docking screening was performed using the Lamarchian genetic algorithm $(\mathrm{GA})^{27}$ and the default parameters were set. The docking model was derived from 100 different runs and the maximum of 250,000 energy evolutions. Finally, the model with lowest binding energy was chosen. In the model with the lowest binding energy, the position of the phosphate group aligns closely with that of the formate and three water molecules in the crystalized structure ${ }^{5,21}$. This model is consistent with the acetyl-phostate docking structure built by Cheung et $\mathrm{al}^{5}$ as shown in supplemental Figure S1. Thus, the model with the lowest binding energy was used as the initial enzyme-substrate complex system. Then for the complex system, the protonate states of all amino acids were validated using MolProbity ${ }^{28}$ and solvated a box of TIP3P water molecules. ${ }^{29} \mathrm{The} \mathrm{X}, \mathrm{Y}$ and Z-axis sizes were determined as $68.095 \AA, 67.882 \AA$ and $73.532 \AA$ respectively by setting the closest distance between any atom of the solute and the edge of the periodic box at $18.0 \AA$. Thus the solvated complex system containing 32,135 atoms was obtained as the initial reaction system in solvate.

\section{Equilibration and optimization of the initial reaction system}

First the solvent was optimized until the root mean squire of energy gradient was less than $1.0 \mathrm{kcal} \mathrm{mol}^{-1} . \AA^{-1}$. Then the reaction system was equilibrated with $2.1 \mathrm{~ns}$ molecular 
dynamics simulation at $298 \mathrm{~K}$ with step-by-step released processes, i.e. $100 \mathrm{ps}$ for NVT + 1.0ns for NPT with constraints $+1.0 \mathrm{~ns}$ for NPT without constraints. Finally, a $10 \mathrm{~ns}$ NPT molecular dynamics simulation was performed without any restraints. Here, all molecular dynamics simulations were run using ACEMD software on $\mathrm{GPU}^{30} .1$ bar pressure was added according to Berendsen method and the temperature is controlled at $298 \mathrm{~K}$ by a Langevin thermostat. All bonds had been constrained using SHAKE and the integration time step was $4 \mathrm{fs}$ with amber99 force field ${ }^{31}$. In the last $10 \mathrm{~ns}$ molecular dynamics, the system was equilibrated and the RMSD of molecule dynamics trajectory using the fluctuation of the $\mathrm{Ca}$ atoms was shown in supplemental Figure S2. In which the largest cluster of RMSD distribution was when RMSD $=2.1 \AA$ as shown in the supplemental panel of Figure S2.

We selected one representative conformation from the final $10 \mathrm{~ns}$ trajectory based on the largest cluster $(\mathrm{RMSD}=2.1 \AA)$ and the interaction distances between the oxygen atom of water molecule and phosphate atom of the substrate should be suitable for a nucleophilic attack because the water molecule has a nucleophilic attack to the phosphate group as validated previously ${ }^{4-5,21}$. Here the distance for nucleophilic attack was marked as $d_{1}$ as shown in Figure 2a. The snapshot at 7.70 ns was chosen since the $d_{1}$ value was equivalent roughly to its average value in all conformations. The interactions between the substrate and its surrounding residues (V14, G16, V17, G18, F19, R20, W21 and N38) are shown in Figure 2a marked by purple dash lines. Using the configuration at $7.70 \mathrm{~ns}$ as the equilibrated enzymatic system, a set of active atoms were defined including all solute atoms and the solvent molecules within $18.0 \AA$ from the center of the active site. The other atoms were fixed as a preferred approximation of the boundary ${ }^{32}$. Here we used the oxygen 
atom of crystal water as the center of the active site.

\section{QM/MM model}

Based on the interaction between the substrate and enzyme environment, the QM part was chosen and consisted of 55 atoms including the benzoyl phosphate, one crystal water molecule, the side chain of Arg20, the main chain of Val14 and the side chain of Asn38 as shown Figure 2a. The rest formed the MM part. The QM part has been described by ab initio density functional models with Gaussian software ${ }^{33}$ at the levels of B3LYP/6-31G*34${ }^{36}$, and the MM part was run using TINKER software ${ }^{37}$ with the AMBER99 force field ${ }^{31}$ and the TIP3P model for water molecules ${ }^{29}$. The QM/MM nonbonded interactions have been treated as described in detail by Field and Karplus et $\mathrm{al}^{38}$ and the bonded boundaries were treated by the improved pseudobond approach ${ }^{39}$.

\section{Calculating multi-dimensional potential energy surfaces (PESs)}

Starting from the representative equilibrated conformation (Figure 2a), we computed the two-dimensional PESs to explore the possible catalytic mechanisms by multidimensional reaction coordinate driving ${ }^{40-41}$. The approach has been successfully applied to more than one enzyme systems ${ }^{40-45}$. Every energy point on two-dimensional PESs along the reaction coordinates was calculated by geometry optimization at the B $3 \mathrm{LYP} / 6-31 \mathrm{G}^{*}$ level ${ }^{34,46-47}$ of theory with the corresponding restraints of the reaction coordinate. Here the restraint of the reaction coordinate was performed by using a harmonic restraining potential at the $j$-th point i.e. $V_{\text {restrain }}=\frac{1}{2} k_{\text {restrain }}\left(R_{c}^{j}-R_{c}^{j}(r e f)\right)^{2}$. In the equation, $R_{c}$ is defined as a combination of interatomic Euclidean distances associated with forming/breaking covalent bonds in the $c$-th dimension of the concerned mechanism, namely, $R_{c}=\sum b_{i} d_{i}$, in which $d_{i}$ is the interatomic distance, with $b_{i}=1$ if the bond is to be broken or $b_{i}=-1$ 
if the bond is to be formed. The restraining force constant $k_{\text {restrain }}$ is $1000 \mathrm{kcal} . \mathrm{mol}^{-1} . \AA^{-2}$. $R_{c}^{\mathrm{j}}(r e f)$ is the $j$-th point of the $c$-th dimension of reference reaction coordinate value and is increased by $0.1 \AA$ after one point has been optimized to optimize the next point. The actual reaction coordinate values of every point after the restrained optimization are always within $0.01 \AA$ of the respective reference value. To explore two-dimensional potential energy surface, we used the first dimension to dedicate the nucleophilic attacking of crystal water. Thus, the first dimension of reaction coordinate $(c=1)$ was defined as $R_{1}=d_{1}-$ $d_{2}$, where $d_{1}$ described the distance between the oxygen atom of the water molecule and the phosphorus atom of the substrate, and $d_{2}$ described the distance between the phosphorus atom and the oxygen atom of the carboxyl group of the substrate as shown in Figure 2a. The drop of the $R_{c}(r e f)$ value means the nucleophilic attack occurs between the oxygen atom of water molecule and the phosphorus atom of the substrate. The second dimension of the reaction coordinate is to show the transfer of a proton of the water molecule. To validate the role of Asn38, we set up two sets of the second-dimension reaction coordinates $(c=2)$, one of which is involving Asn38. In this dimension of reaction coordinates, the interatomic distances were marked as $d_{3}[\mathrm{Ow}-\mathrm{Hw}], d_{4}[\mathrm{Hw}-\mathrm{O} 2]$ and $d_{5}$ [On-Hw], which respectively mark the distance between the oxygen atom and the hydrogen atom of the crystal water, the distance between the hydrogen atom of crystal water and the oxygen atom of phosphate, and the distance between the carbonyl oxygen atom of the side chain of Asn38 and the hydrogen atom of crystal water as shown in Figure 2a. When Asn38 participates in the bond-forming/breaking catalytic process, the seconddimension reaction coordinate is defined as $R_{2}=d_{4}+d_{5}-d_{3}$. During this process, the proton is transferred to the oxygen atom of the phosphate group with the help of Asn38. 
Otherwise, the second-dimension reaction coordinate is defined as $R_{2}=d_{4}-d_{3}$, where Asn38 is just catalytic environment, which means the proton is directly transferred to the oxygen atom of the phosphate group. Following the 2D reaction coordinate driving, we explored the PESs.

From the initial value $d_{1}=3.60 \AA, d_{2}=1.97 \AA, d_{3}=1.00 \AA, d_{4}=2.95 \AA$ and $d_{5}=$ $1.76 \AA$ shown in Figure 2a, the optimizations were performed with the constraints of specific $R_{1}$ and $R_{2}$ values step-by-step until the formation and breaking of the bonds were finished both at both the first dimension and the second dimension.

\section{Results and Discussion}

We will first describe the obtained PESs including their chemical mechanisms with reaction details. Then, we presented the mechanism-exploring results including the nucleophilic attack and the carbonyl-assisted mechanism following the hydrogen transfer. Finally, we analyze how transition states have been stabilized by the enzyme environments.

\section{A detailed catalytic mechanism}

Figure 3a shows a two-dimensional PES with the reaction coordinate: $R_{1}=d_{1}-d_{2}$; $R_{2}=d_{4}+d_{5}-d_{3}$. Based on this PES, the minimum energy surface (marked red line, Scheme 3) has a transition state (TS1) with an energy barrier (14.60 kcal mol $\left.l^{-1}\right)$, which is in agreement with the experimental data $\left(14.76 \mathrm{kcal} \mathrm{mol}^{-1}\right)^{5}$. As shown in this figure, Scheme 1 and 2 are not preferred reaction schemas.

Using the equilibrated conformation (Figure 2a) as the starting, following the minimum energy pathway (Figure 3a, red line), the Oxygen atom (Ow) of crystal water engaged in a nucleophilic attack with the phosphorus atom (P), ultimately combining to 
form TS1. More specifically, the Oxygen atom $(\mathrm{Ow})$ approached the phosphorus atom $(\mathrm{P})$ step-by-step, reaching the point where $d_{1}=3.21 \AA, d_{2}=1.97 \AA$, which is a local energy minimum of the system. The local energy minimum is used as the reactant state (RS) point (Figure $2 \mathrm{~b}$ and $3 \mathrm{a}$ ). At this stage, the $2 \mathrm{nd}$ dimensional reaction coordinates composed of three chemical bonds $\left(d_{3}=1.00 \AA, d_{4}=2.74 \AA\right.$ and $d_{5}=1.74 \AA$, Figure $\left.2 \mathrm{~b}\right)$ did not have too much change compared to the equilibrated conformation (Figure 2a). We noted the crystal water was strongly stabilized in the enzyme environment by the interaction $\left(d_{5}=1.74 \AA\right)$ between the oxygen atom of the carbonyl group at the side chain of Asn38 and the hydrogen atom of the crystal water molecule. This interaction was consistent with that described in others' papers published about crystal structures and experimental data ${ }^{4,16}$.

In the first dimension, from RS to TS1, the bond [P-O1] was broken when the bondlength changes from $d_{2}=1.97$ to $3.03 \AA$ and the bond [Ow-P] formed along with the change of $d_{1}$ from 3.21 to $2.11 \AA$. The three reaction bonds in the second dimension show very little change from RS to TS1. Up to TS1, an energy barrier occurs (Figure 3a), where $d_{1}=$ $2.11 \AA, d_{2}=3.03 \AA, d_{3}=1.04, d_{4}=2.41 \AA$ and $d_{5}=1.56 \AA$ (Figure $2 \mathrm{c}$ ). Importantly, the change of reaction coordinates shows that the dominant reaction process occurs. That is to say, the nucleophilic attack is almost finished when the bond between the Oxygen atom $(\mathrm{Ow})$ of crystal water and the phosphorus atom (P) forms. In next part, we will analyze TS1 in detail.

Following the nucleophilic attack, the transfer process of the hydrogen atom occurs along the minimum energy pathway. When the energy decreases, the hydrogen atom from the crystal water molecule is translated to the phosphate group spontaneously. To reveal the transfer of the hydrogen atom, we decomposed the $R_{1}$ and $R_{2}$ along the minimum 
energy pathway (Figure 3a, red line). $d_{1}$ changed from 2.11 to $1.70 \AA$ between TS1 and PS (Figure 4a), and the formed bond [Ow-P] becomes more stable. The change in $d_{2}$ from 3.03 to $3.83 \AA$ means the benzoyl group entirely drops out. When $d_{3}$ changes from 1.04 to $2.47 \AA$, the hydrogen atom was transferred out as donor, correspondingly, when $d_{4}$ changes from 2.49 to $1.03 \AA$, the hydrogen atom is transferred to the atom O2. The change of $d_{5}[\mathrm{On}-\mathrm{Hw}]$ is particularly interesting. $d_{5}$ first decreased from 1.56 to $1.0 \AA$, which means the hydrogen atom is transferred to the carbonyl of Asn 38 ; then the value of $d_{5}$ from 1.0 to $1.45 \AA$ meaning that the hydrogen atom was then transferred to the destination (O2). This process means the hydrogen atom was not transferred directly from the donor to the receptor, but followed a carbonyl-assisted shuttle mechanism. In the next part, we will analyze this shuttle mechanism in more detail. At PS, the five distances were $d_{1}=1.70 \AA$, $d_{2}=3.83 \AA, d_{3}=2.47 \AA, d_{4}=1.03 \AA$ and $d_{5}=1.45 \AA$ (Figure $2 \mathrm{~d}, 3 \mathrm{a}$ and $4 \mathrm{a}$ ), which means the new bonds were formed and the expected bonds were broken. Thus, the product was achieved following the reaction pathway. Comparatively speaking, Scheme 1 and 2 showed an energy barrier that was too high, which is unrealistic in fulfilling the catalysis process.

To further validate the role of Asn38, we also calculated one two-dimensional PES with the reaction coordinate: $R_{1}=d_{1}-d_{2} ; R_{2}=d_{4}-d_{3}$, where Asn38 is used as the catalytic environment. Based on the potential energy surface, there are two reaction pathways (Scheme 1' and Scheme 3'), which are shown in Figure 3b.

In Scheme 1' (Figure 3b), the hydrogen atom of the crystal water molecule was first transferred to phosphate group across a transition state (TS2) with a low barrier of 10.60 $\mathrm{kcal} \mathrm{mol}^{-1}$. At TS2, the proton transfer formed the transition state. At IM1, there is a very stable intermediate state with a lower energy valley at the energy surface. The nucleophilic 
attacking occurred along Scheme 1' from IM1 to PS. In this step, another transition state (marked as TS3) with a higher energy barrier of $29.58 \mathrm{kcal} \mathrm{mol}^{-1}$ needed to be overcome.

In Scheme 3' (Figure 3b), the minimum energy pathway occurred across a transition state $\left(\mathrm{TS1} 1^{\prime}\right)$ with an energy barrier of $14.30 \mathrm{kcal} \mathrm{mol}^{-1}$. The pathway is very similar to Scheme 3 in Figure 3a. The subtle difference is that $d_{5}=1.60 \AA$ in Scheme $3^{\prime}$ at TS1' is slightly larger than $d_{5}=1.56 \AA$ in Scheme 3 at TS1. Undoubtedly, these distances both means one strong interaction with the crystal water molecule. It is worth nothing that a shuttle transfer mechanism was shown as Figure 4a. But in Figure 4b, at the PS, a pseudoPS state was obtained because we saw the transferring hydrogen atom was bonded with the carbonyl of $\operatorname{Asn} 38\left(d_{5}=1.00 \AA\right)$ but the oxygen atom of phosphate group $\left(d_{4}=1.50 \AA\right)$ as shown in Figure 4b. It is easy to understand for that because the hydrogen atom moves to the carbonyl of Asn38 spontaneous, but cannot bond to the oxygen atom of phosphate group without any restrain force to overcome the transferring energy barrier. It was also shown that the Asn38-involved reaction coordinate is preferred to be used as the intrinsic reaction coordinate $\mathrm{e}^{48}$. Our strategy of using the sets of reaction coordinates also means that the right reaction coordinate to be chosen is key in the complex reaction system ${ }^{49-50}$.

Totally, based on the two sets of reaction coordinates and their corresponding PESs, a preferable reaction mechanism was achieved as (Figure 3a, Scheme 3). Next part, we will focus on the analysis of reaction mechanism based on the obtained MEP (Figure 3a, Scheme 3).

\section{The nucleophilic attack mechanism in rate-limiting step}

As described in the last part, following the nucleophilic attack (Figure 4a), when $d_{1}$ changes from 3.21 to $2.61 \AA, d_{2}$ gradually increases; however, when $d_{1}$ changes from 
2.51 to $2.11 \AA, d_{2}$ increases rapidly from 2.13 to $3.03 \AA$. As a whole attack mechanism, this process is a loose associated reaction process for the formation of the bond [Ow-P] and the breaking of the bond [P-O1]. The other three reaction coordinates $\left(d_{3-5}\right)$ had very little change till TS1. Along the MEP, there was a higher energy barrier $\left(14.60 \mathrm{kcal} \mathrm{mol}^{-1}\right)^{5}$ at TS1. So TS1 is the rate-limited transition state. Here $d_{1}=2.11 \AA, d_{2}=3.03 \AA$ and $d_{3}=1.04$ $\AA$; these 3 bonds formed a loose associated translation state and the transfer of the hydrogen atom did not occur. From TS1 to PS, the reaction energy steeply decreases, and at PS, the bond [P-O1] had a bond length of $3.83 \AA$, which means the bond was broken. Also at PS, the bond [Ow-P] has a length of $1.70 \AA$, indicating that the nucleophilic attack is finished.

\section{The carbonyl-assistant mechanism in hydrogen-translated step.}

After the nucleophilic attack comes to finish as shown in Figures 2c and 3a, the hydrogen atom $(\mathrm{Hw})$ of the crystal water molecule as an electrophilic atom will be transferred to an electron-rich group, achieving the stable product state. Starting from the TS1, the bond [Ow-Hw] starts to increase. When $d_{3}[\mathrm{Ow}-\mathrm{Hw}]$ changes from 1.06 to 1.51 $\AA, d_{4}[\mathrm{O} 2-\mathrm{Hw}]$ has no significant change, but $d_{5}[\mathrm{On}-\mathrm{Hw}]$ sharply decreases from 1.45 to $1.01 \AA$. Thus, the change means the $\mathrm{Hw}$ atom first transfers to the carbonyl oxygen atom $(\mathrm{On})$ and the electrophilic group transfers to its destination $(\mathrm{O} 2)$ indirectly. Then, the hydrogen atom $(\mathrm{Hw})$ is transferred from the On to the $\mathrm{O} 2$ atom as shown in Figure 4a, where $d_{5}$ changes from 1.02 to $1.45 \AA$ and $d_{4}$ changes from 1.82 to $1.03 \AA$ until it finally reaches the PS. By this indirect process, the $\mathrm{Hw}$ atom is transferred to the $\mathrm{O} 2$ with the assistance of the carbonyl oxygen atom (On) of Asn38. The carbonyl-assistant mechanism facilitates the occurrence of the reaction and improves the reaction efficiency as shown in a similar reaction presented by Rizzi et.al ${ }^{51}$. Compared to the non-enzyme environment, 
where there is no shuttle mechanism involved, the hydrogen atom is directly transferred to the oxygen atom of the phosphate group from the water molecule. This direct transfer may be one of the reasons why the energy barrier is too high, for example the catalytic ability is $10^{-20} \mathrm{~S}^{-1}$ in the non-enzyme environment as shown in the experimental data ${ }^{52}$ and the theoretic calculation ${ }^{53}$.

\section{The strategy for Transition state stabilization utilized by the enzyme environment}

Above, we analyzed a nucleophilic-attack and the ensuing carbonyl-assisted shuttle mechanism for the catalytic process. Here we analyze the stabilization strategy of the transition state. The transition state (TS1) with the energy barrier $14.60 \mathrm{kcal} \mathrm{mol}^{-1}$ was stabilized by the enzyme environment. Asn38 participates in the catalytic reaction by a carbonyl-assistant trick in the catalytic center. Figure $2 \mathrm{c}$ shows the interaction network around the active site and Figure 5 shows the details of the hydrogen-bond interaction specifically at TS1. A conserved amino acid peptide (consisting of V14, G16, V17, G18, F19, R20 and W21) and the N38 form a binding pocket, which embraces the substrate as shown by the amino acids (in green) and the hydrogen-bond interaction (in purple) in Figure 2c. In Figure 5, the dash lines show the detailed hydrogen bonding between the enzyme environment and the substrate. In G16, V17, G18, F19, the hydrogen bonds take place at the Nitrogen atom of the main chain. In R20 and W21, the nitrogen atoms of the side chain provide the hydrogen-bond interaction. Simultaneously, the carbonyl group of V14 and the amide group of N38 have the interactions with the crystal water molecule at the bottom of the binding pocket. The interaction between the crystal water and Asn38 is notably stronger $\left(d_{5}=1.56 \AA\right.$, Figure $\left.2 \mathrm{c}\right)$ than the other non-bond interaction. The interaction between the substrate and Asn38 highly stabilized the conformation state and 
provided the foundation for carrying out a carbonyl-assisted mechanism. The R20 and W21 did not directly interact with the catalytic center, however they both interact with the substrate across a solvent water molecule bridge. We also noted there was a R20-G91 saltbridge interaction, which is a very interesting interaction as discussed in the paper by Sonia et $\mathrm{al}^{54}$. The carboxyl group of G91 also forms the hydrogen-bond interactions with the main-chain nitrogen atom of Asn38. These results are agreeable with the experimental result ${ }^{54}$, which provided the interaction network with the active site contributing to the enzyme activity.

\section{Conclusions}

We have achieved a detailed, feasible nucleophilic-attack and its ensuing carbonylassisted catalytic mechanism as well as an experiment-agreeable energy barrier for acetylphosphatase. In so doing, we have revealed new insights into the chemical mechanism: the nucleophilic attack of water molecule is a loose associated process along the formation of the $[\mathrm{Ow}-\mathrm{P}]$ bond and the breaking of the [P-O1] bond. During the attacking, the rate-limiting barrier arises because the nucleophilic attack takes place while a hydrogen atom of crystal water molecule remains. The subsequent step is energetically a downhill process because the hydrogen atom is transferred spontaneously with a carbonyl-assisted mechanism from Asn38. As a whole process, the carbonyl group assisted the chemical mechanism by forming the strong interaction and shuttling a transferring hydrogen atom.

Besides the chemical mechanism, PhAcP provided a well-organized enzymatic environment to specifically stabilize the rate-limiting transition state (TS1). At TS1, the peptide interacts with the phosphate group of the substrate, and increased the localization 
of the phosphate atom. Simultaneously, the Asn38 has a close-distance interaction with the substrate and localized the charge of the crystal water molecule by redistributing the more negative charge on the oxygen atom of the crystal water molecule. The transition state stabilization strategy is from the charge distribution by the direct interaction network around the catalytic center. The salt-bridge from G91 was also found to stabilize the catalytic environment by forming the hydrogen-bond interaction with the catalytic residue Asn38 and Arg20.

In sum, we have employed a QM/MM energy function to study the reaction mechanism of ACP, and we have gathered the new insights into the nucleophilic-attack-induced carbonyl-assisted catalytic mechanism and the transition state stabilization strategy based on an enzymatic network contribution. The detailed mechanism will contribute to the design of new catalysis for the development of better biotech enzyme and contribute to the disease-involved study for the ACP-involved signal pathway.

\section{Acknowledgement}

We thank Prof. Haiyan Liu (USTC) for helpful discussions and computing resources provided.

\section{Reference}

1. Pastore, A.; Saudek, V.; Ramponi, G.; Williams, R. J. P., 3-Dimensional Structure of Acylphosphatase Refinement and Structure-Analysis. J Mol Biol 1992, 224 (2), 427440. 
2. Elia, F.; Cantini, F.; Chiti, F.; Dobson, C. M.; Bemporad, F., Direct Conversion of an Enzyme from Native-like to Amyloid-like Aggregates within Inclusion Bodies. Biophys J 2017, 112 (12), 2540-2551.

3. Nassi, P.; Liguri, G.; Nediani, C.; Taddei, N.; Ramponi, G., Increased acylphosphatase levels in erythrocytes, muscle and liver of tri-iodothyronine treated rabbits. Hormone and metabolic research $=$ Hormon- und Stoffwechselforschung $=$ Hormones et metabolisme 1990, 22 (1), 33-7.

4. Stefani, M.; Taddei, N.; Ramponi, G., Insights into acylphosphatase structure and catalytic mechanism. Cell Mol Life Sci 1997, 53 (2), 141-151.

5. Cheung, Y. Y.; Lam, S. Y.; Chu, W. K.; Allen, M. D.; Bycroft, M.; Wong, K. B., Crystal structure of a hyperthermophilic archaeal acylphosphatase from Pyrococcus horikoshii - Structural insights into enzymatic catalysis, thermostability, and dimerization. Biochemistry-Us 2005, 44 (12), 4601-4611.

6. Stefani, M.; Ramponi, G., Acylphosphate phosphohydrolases. Life Chemistry Reports 1995, 12, 271-301.

7. Nassi, P.; Nediani, C.; Liguri, G.; Taddei, N.; Ramponi, G., Effects of Acylphosphatase on the Activity of Erythrocyte-Membrane Ca-2+ Pump. J Biol Chem 1991, $266(17), 10867-10871$.

8. $\quad$ Liguri, G.; Cecchi, C.; Latorraca, S.; Pieri, A.; Sorbi, S.; DeglInnocenti, D.; Ramponi, G., Alteration of acylphosphatase levels in familial Alzheimer's disease fibroblasts with presenilin gene mutations. Neurosci Lett 1996, 210 (3), 153-156. 9. Degl'Innocenti, D.; Ramazzotti, M.; Sarchielli, E.; Monti, D.; Chevanne, M.; Vannelli, G. B.; Barletta, E., Oxadiazon affects the expression and activity of aldehyde 
dehydrogenase and acylphosphatase in human striatal precursor cells: A possible role in neurotoxicity. Toxicology 2019, 411, 110-121.

10. Wijma, H. J.; Floor, R. J.; Jekel, P. A.; Baker, D.; Marrink, S. J.; Janssen, D. B., Computationally designed libraries for rapid enzyme stabilization. Protein engineering, design \& selection : PEDS 2014, 27 (2), 49-58.

11. Plakoutsi, G.; Bemporad, F.; Monti, M.; Pagnozzi, D.; Pucci, P.; Chiti, F., Exploring the mechanism of formation of native-like and precursor amyloid oligomers for the native acylphosphatase from Sulfolobus solfataricus. Structure 2006, 14 (6), 9931001.

12. Saudek, V.; Williams, R. J. P.; Ramponi, G., The Structure and Properties of Horse Muscle Acylphosphatase in Solution - Mobility of Antigenic and Active-Site Regions. Febs Lett 1989, 242 (2), 225-232.

13. Satchell, D. P.; Spencer, N.; White, G. F., Kinetic Studies with Muscle Acylphosphatase. Biochim Biophys Acta 1972, 268 (1), 233-\&.

14. Taddei, N.; Stefani, M.; Vecchi, M.; Modesti, A.; Raugei, G.; Bucciantini, M.; Magherini, F.; Ramponi, G., Arginine-23 Is Involved in the Catalytic Site of Muscle Acylphosphatase. Bba-Protein Struct M 1994, 1208 (1), 75-80.

15. Taddei, N.; Magherini, F.; Chiti, F.; Bucciantini, M.; Raugei, G.; Stefani, M.; Ramponi, G., C-terminal region contributes to muscle acylphosphatase three-dimensional Structure stabilisation. Febs Lett 1996, 384 (2), 172-176.

16. Taddei, N.; Stefani, M.; Magherini, F.; Chiti, F.; Modesti, A.; Raugei, G.; Ramponi, G., Looking for residues involved in the muscle acylphosphatase catalytic 
mechanism and structural stabilization: Role of Asn41, Thr42, and Thr46. BiochemistryUs 1996, 35 (22), 7077-7083.

17. Yeung, R. C. Y.; Lam, S. Y.; Wong, K. B., Crystallization and preliminary crystallographic analysis of human common-type acylphosphatase. Acta Crystallogr F 2006, $62,80-82$.

18. Cheung, Y. Y.; Allen, M. D.; Bycroft, M.; Wong, K. B., Crystallization and preliminary crystallographic analysis of an acylphosphatase from the hyperthermophilic archaeon Pyrococcus horikoshii. Acta Crystallogr D 2004, 60, 1308-1310.

19. Zuccotti, S.; Rosano, C.; Ramazzotti, M.; Degl'Innocenti, D.; Stefani, M.; Manao, G.; Bolognesi, M., Three-dimensional structural characterization of a novel Drosophila melanogaster acylphosphatase. Acta Crystallogr D 2004, 60, 1177-1179.

20. Rosano, C.; Zuccotti, S.; Bucciantini, M.; Stefani, M.; Ramponi, G.; Bolognesi, M., Crystal structure and anion binding in the prokaryotic hydrogenase maturation factor HypF acylphosphatase-like domain. J Mol Biol 2002, 321 (5), 785-796.

21. Thunnissen, M. M. G. M.; Taddei, N.; Liguri, G.; Ramponi, G.; Nordlund, P., Crystal structure of common type acylphosphatase from bovine testis. Structure 1997, 5 (1), 69-79.

22. Degl'Innocenti, D.; Ramazzotti, M.; Marzocchini, R.; Chiti, F.; Raugei, G.; Ramponi, G., Characterization of a novel Drosophila melanogaster acylphosphatase. Febs Lett 2003, 535 (1-3), 171-174.

23. Corazza, A.; Rosano, C.; Pagano, K.; Alverdi, V.; Esposito, G.; Capanni, C.; Bemporad, F.; Plakoutsi, G.; Stefani, M.; Chiti, F.; Zuccotti, S.; Bolognesi, M.; Viglino, 
P., Structure, conformational stability, and enzymatic properties of acylphosphatase from the hyperthermophile Sulfolobus solfataricus. Proteins 2006, 62 (1), 64-79.

24. Fiaschi, T.; Marzella, R.; Veggi, D.; Marzocchini, R.; Raugei, G.; Rocchi, M.; Ramponi, G., Assignment of the human erythrocyte acylphosphatase gene (ACYP1) to chromosome band 14q24.3. Cytogenet Cell Genet 1998, 81 (3-4), 235-236.

25. Sadowski, J.; Gasteiger, J.; Klebe, G., Comparison of Automatic ThreeDimensional Model Builders Using 639 X-ray Structures. Journal of Chemical Information and Modeling 1994, 34 (4), 1000-1008.

26. Morris, G. M.; Goodsell, D. S.; Halliday, R. S.; Huey, R.; Hart, W. E.; Belew, R. K.; Olson, A. J., Automated docking using a Lamarckian genetic algorithm and an empirical binding free energy function. J Comput Chem 1998, 19 (14), 1639-1662.

27. Goodsell, D. S.; Morris, G. M.; Olson, A. J., Automated docking of flexible ligands: Applications of AutoDock. J Mol Recognit 1996, 9 (1), 1-5.

28. Chen, V. B.; Arendall, W. B., 3rd; Headd, J. J.; Keedy, D. A.; Immormino, R. M.; Kapral, G. J.; Murray, L. W.; Richardson, J. S.; Richardson, D. C., MolProbity: all-atom structure validation for macromolecular crystallography. Acta crystallographica. Section D, Biological crystallography 2010, 66 (Pt 1), 12-21.

29. Jorgensen, W. L.; Chandrasekhar, J.; Madura, J. D.; Impey, R. W.; Klein, M. L., Comparison of simple potential functions for simulating liquid water. J Chem Phys 1983, $79(2), 926$.

30. Harvey, M. J.; Giupponi, G.; Fabritiis, G. D., ACEMD: Accelerating Biomolecular Dynamics in the Microsecond Time Scale. Journal of Chemical Theory and Computation 2009, 5 (6), 1632-1639. 
31. Cornell, W. D.; Cieplak, P.; Bayly, C. I.; Gould, I. R.; Merz, K. M.; Ferguson, D. M.; Spellmeyer, D. C.; Fox, T.; Caldwell, J. W.; Kollman, P. A., A Second Generation Force Field for the Simulation of Proteins, Nucleic Acids, and Organic Molecules. $J$ Am Chem Soc 1995, 117 (19), 5179-5197.

32. Xie, L.; Liu, H.; Yang, W., Adapting the nudged elastic band method for determining minimum-energy paths of chemical reactions in enzymes. J Chem Phys 2004, 120 (17), 8039-52.

33. Frisch, M. J. T., G. W.; Schlegel, H. B.; Scuseria, G. E.; Robb, M. A.; Cheeseman, J. R.; Montgomery, J. A., Jr.; Vreven, T.; Kudin, K. N.; Burant, J. C.; Millam, J. M.; Iyengar, S. S.; Tomasi, J.; Barone, V.; Mennucci, B.; Cossi, M.; Scalmani, G.; Rega, N.; Petersson, G. A.; Nakatsuji, H.; Hada, M.; Ehara, M.; Toyota, K.; Fukuda, R.; Hasegawa, J.; Ishida, M.; Nakajima, T.; Honda, Y.; Kitao, O.; Nakai, H.; Klene, M.; Li, X.; Knox, J. E.; Hratchian, H. P.; Cross, J. B.; Bakken, V.; Adamo, C.; Jaramillo, J.; Gomperts, R.; Stratmann, R. E.; Yazyev, O.; Austin, A. J.; Cammi, R.; Pomelli, C.; Ochterski, J. W.; Ayala, P. Y.; Morokuma, K.; Voth, G. A.; Salvador, P.; Dannenberg, J. J.; Zakrzewski, V. G.; Dapprich, S.; Daniels, A. D.; Strain, M. C.; Farkas, O.; Malick, D. K.; Rabuck, A. D.; Raghavachari, K.; Foresman, J. B.; Ortiz, J. V.; Cui, Q.; Baboul, A. G.; Clifford, S.; Cioslowski, J.; Stefanov, B. B.; Liu, G.; Liashenko, A.; Piskorz, P.; Komaromi, I.; Martin, R. L.; Fox, D. J.; Keith, T.; Al-Laham, M. A.; Peng, C. Y.; Nanayakkara, A.; Challacombe, M.; Gill, P. M. W.; Johnson, B.; Chen, W.; Wong, M. W.; Gonzalez, C.; Pople, J. A. , Gaussian 03;Gaussian, Inc.: Wallingford, CT 2003. 
34. Vosko, S. H.; Wilk, L.; Nusair, M., Accurate spin-dependent electron liquid correlation energies for local spin density calculations: a critical analysis. Can. J. Phys. 1980, $58(8), 1200-1211$.

35. Becke, A. D., Density-functional thermochemistry. III. The role of exact exchange. J Chem Phys 1993, 98 (7), 5648.

36. Stephens, P. J.; Devlin, F. J.; Chabalowski, C. F.; Frisch, M. J., Ab Initio Calculation of Vibrational Absorption and Circular Dichroism Spectra Using Density Functional Force Fields. The Journal of Physical Chemistry 1994, 98 (45), 11623-11627.

37. Ren, P. Y.; Ponder, J. W., Polarizable atomic multipole water model for molecular mechanics simulation. $J$ Phys Chem B 2003, 107 (24), 5933-5947.

38. Field, M. J.; Bash, P. A.; Karplus, M., A combined quantum mechanical and molecular mechanical potential for molecular dynamics simulations. J Comput Chem 1990, $11(6), 700-733$.

39. Zhang, Y., Improved pseudobonds for combined ab initio quantum mechanical/molecular mechanical methods. J Chem Phys 2005, 122 (2), 024114. 40. Zhang, Y.; Liu, H.; Yang, W., Free energy calculation on enzyme reactions with an efficient iterative procedure to determine minimum energy paths on a combined ab initio QM/MM potential energy surface. J. Chem. Phys. 2000, 112, 3483.

41. Zhang, Y.; Zhao, Z.; Liu, H., Deriving Chemically Essential Interactions Based on Active Site Alignments and Quantum Chemical Calculations: A Case Study on Glycoside Hydrolases. ACS Catalysis 2015, 5 (4), 2559-2572.

42. Levitt, M., Birth and future of multiscale modeling for macromolecular systems (Nobel Lecture). Angew Chem Int Ed Engl 2014, 53 (38), 10006-18. 
43. Senn, H. M.; Thiel, W., QM/MM methods for biomolecular systems. Angew Chem Int Ed Engl 2009, 48 (7), 1198-229.

44. Lei, J.; Zhou, Y.; Xie, D.; Zhang, Y., Mechanistic insights into a classic wonder drug--aspirin. J Am Chem Soc 2015, 137 (1), 70-3.

45. Sirin, G. S.; Zhang, Y., How is acetylcholinesterase phosphonylated by soman? An ab initio QM/MM molecular dynamics study. The journal of physical chemistry. A 2014, $118(39), 9132-9$.

46. Becke, A. D., Density-functional thermochemistry. III. The role of exact exchange. Journal of chemical physics 1993, 98 (7), 5648-5652.

47. Stephens, P. J.; Devlin, F. J.; Chabalowski, C. F.; Frisch, M. J., Ab initio calculation of vibrational absorption and circular dichroism spectra using density functional force fields. J. Phys. Chem. 1994, 98 (45), 11623-11627.

48. Fukui, K., Role of frontier orbitals in chemical reactions. Science 1982, 218 (4574), 747-54.

49. Dickson, B. M.; Makarov, D. E.; Henkelman, G., Pitfalls of choosing an order parameter for rare event calculations. J Chem Phys 2009, 131 (7), 074108.

50. Liu, H. Y.; Zhang, Y. K.; Yang, W. T., How is the active site of enolase organized to catalyze two different reaction steps? J. Am. Chem. Soc. 2000, 122, 6560-6570.

51. Rizzi, G. P., Evidence for an azomethine ylide intermediate in the carbonylassisted decarboxylation of sarcosine. Novel synthesis of dl-phenylephrine hydrochloride. J. Org. Chem. 1970, 35 (6), 2069-2072.

52. Klahn, M.; Rosta, E.; Warshel, A., On the mechanism of hydrolysis of phosphate monoesters dianions in solutions and proteins. J Am Chem Soc 2006, 128 (47), 15310-23. 
53. Branduardi, D.; De Vivo, M.; Rega, N.; Barone, V.; Cavalli, A., Methyl Phosphate Dianion Hydrolysis in Solution Characterized by Path Collective Variables Coupled with DFT-Based Enhanced Sampling Simulations. Journal of Chemical Theory and Computation 2011, 7 (3), 539-543.

54. Lam, S. Y.; Yeung, R. C.; Yu, T. H.; Sze, K. H.; Wong, K. B., A rigidifying saltbridge favors the activity of thermophilic enzyme at high temperatures at the expense of low-temperature activity. PLoS biology 2011, 9 (3), e1001027. 
Figure 1. Proposed reaction schemes.

\section{Scheme 1}

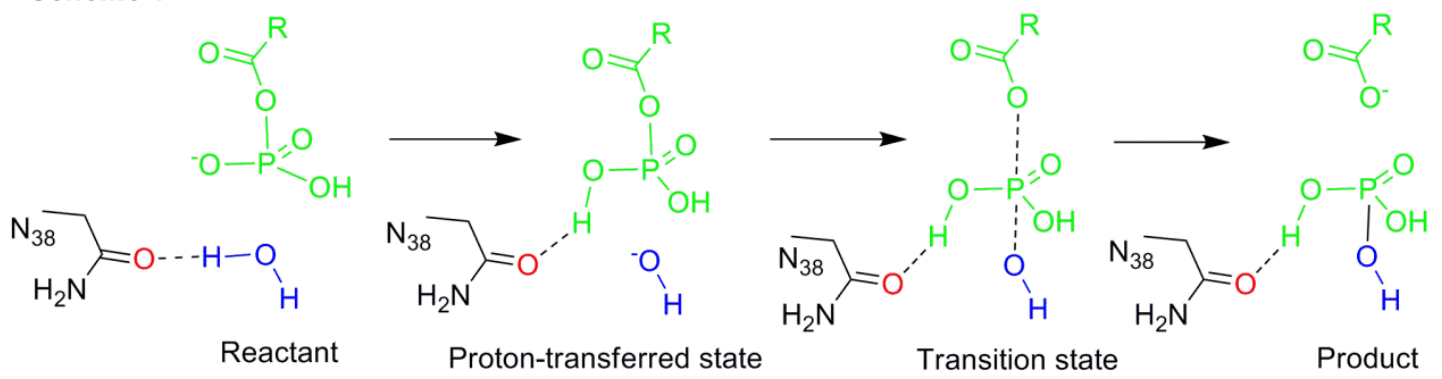

Scheme 2
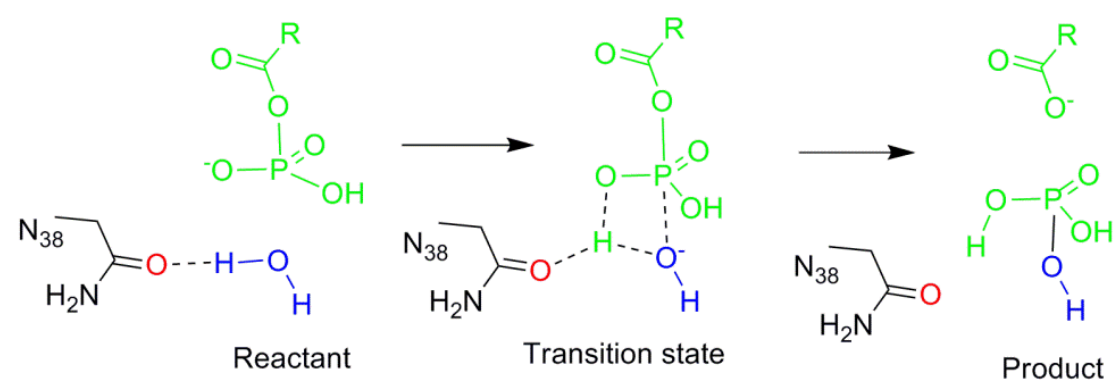

Scheme 3
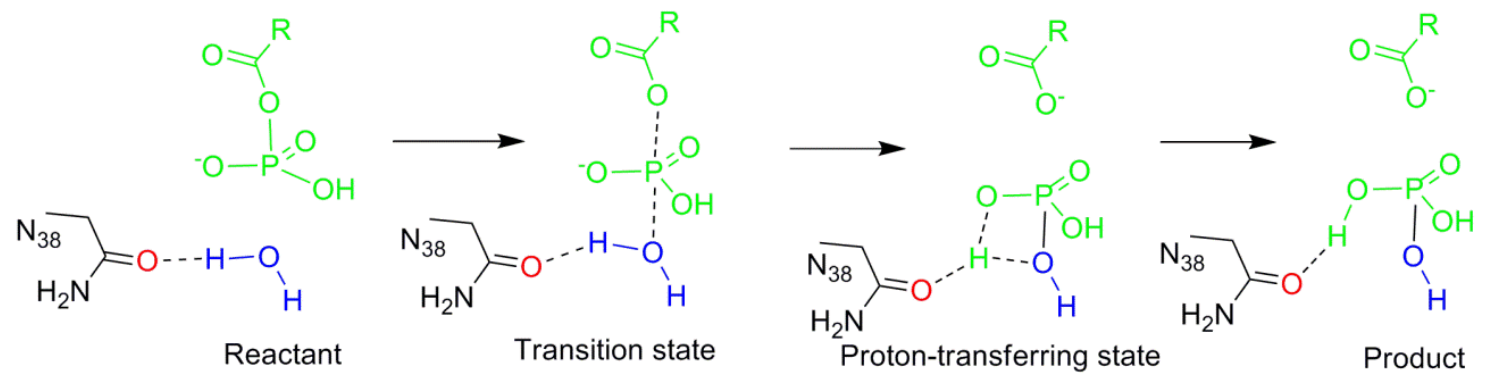

$\mathrm{R}=$ Acetyl or Propionyl or Butyryl and so on. In the paper, $\mathrm{R}=\mathrm{Benzoyl}$ 
Figure 2. The key conformation states for the catalytic mechanism of acylphosphatase. (a) The substrate, the amino acids of catalytic center and the definition of the distances from $d_{1}$ to $d_{5}$ for initial equilibrated complex system; (b) The reactant state(RS); (c) The transition state(TS1); (d) The product state (PS). The distance unit is angstrom.
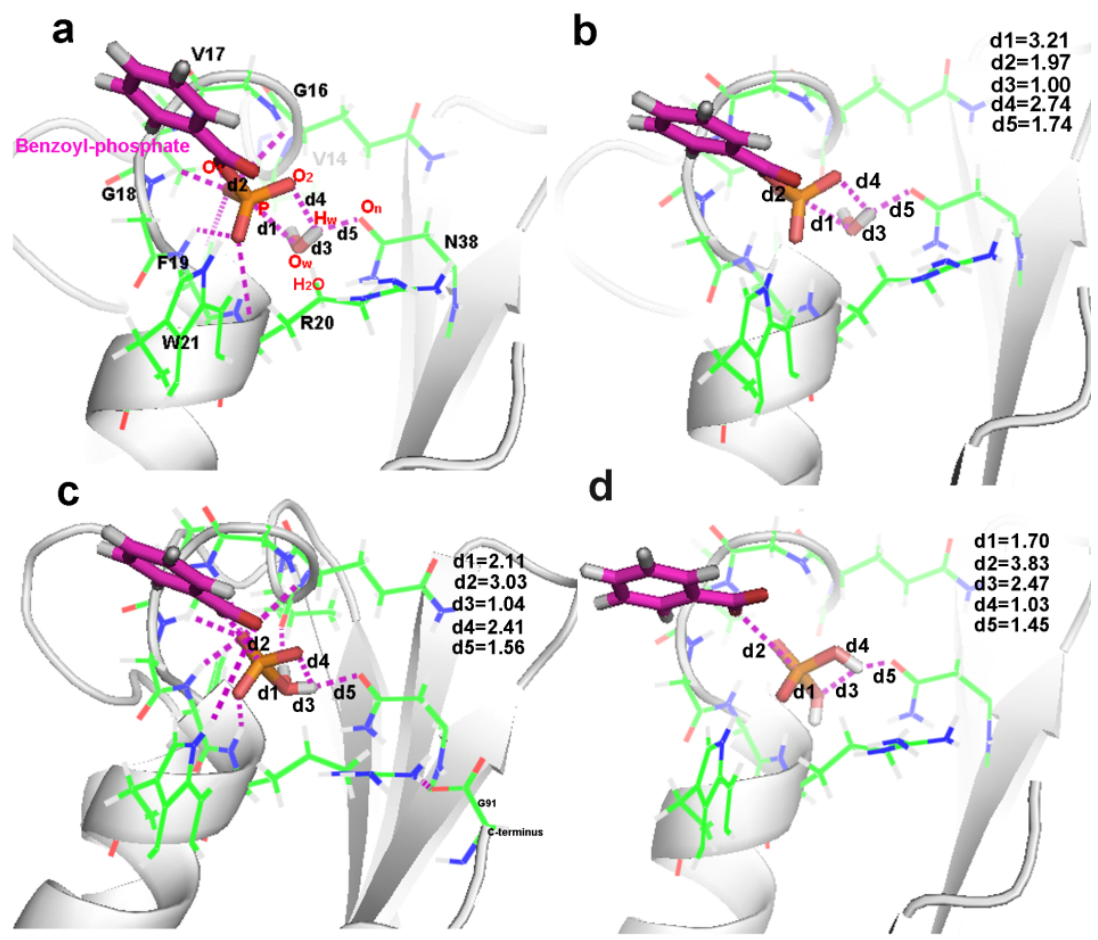
Figure 3. The two-dimensional of potential energy surfaces (PESs). Proposed reaction scheme 1-3 was shown in yellow dash line, blue dash line and red line. (a) The PES with the reaction coordinates: $R_{1}=d_{1}-d_{2} ; R_{2}=d_{4}+d_{5}-d_{3}$. (b) The PES with the reaction coordinates: $R_{1}=d_{1}-d_{2} ; R_{2}=d_{4}-d_{3}$.
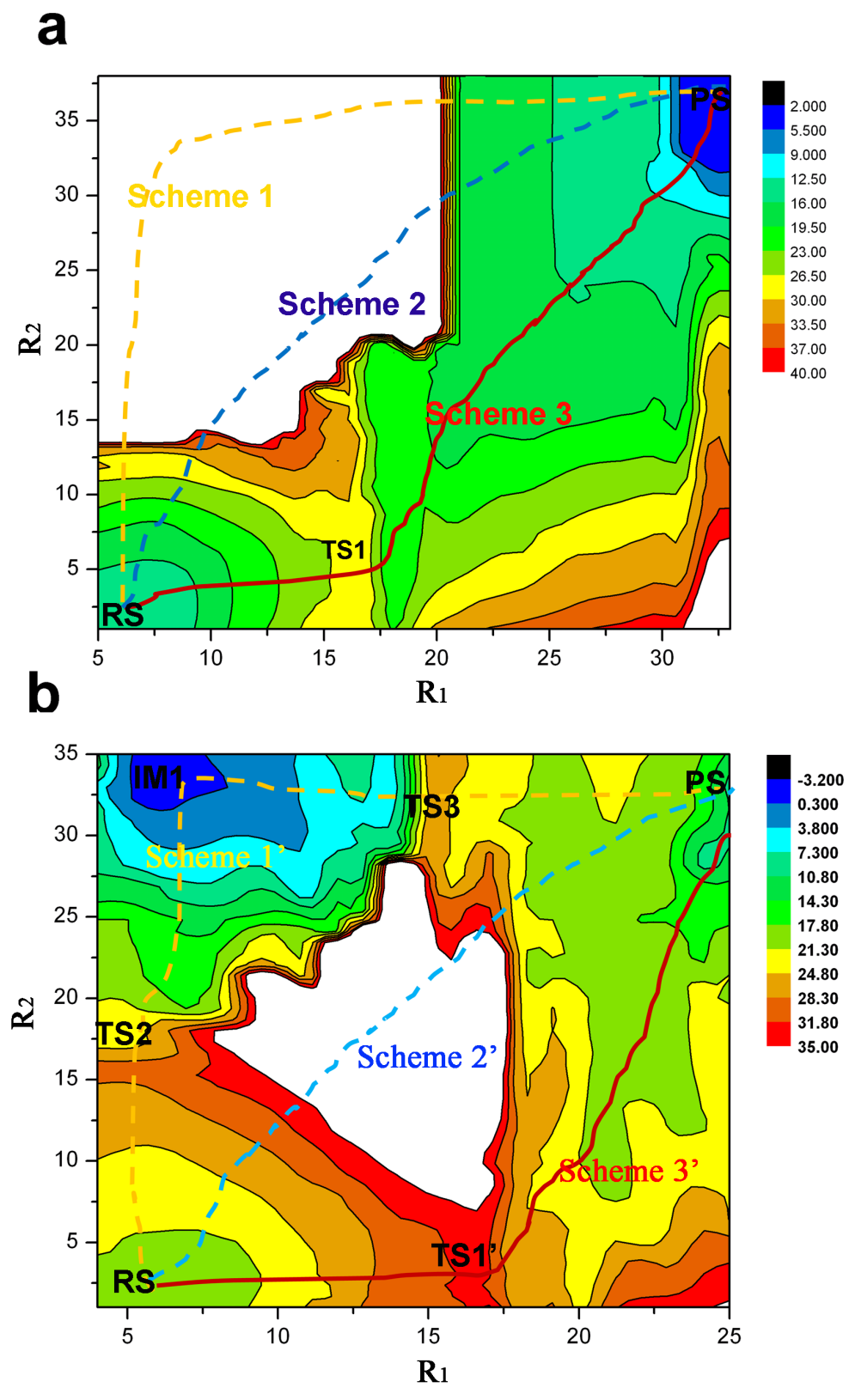
Figure 4. The evolution of the decomposed reaction coordinates along the minimum energy pathway. (a) The bond changes for Scheme3 in Figure 3a. (b) The bond changes for Scheme 3' in Figure 3b.

a
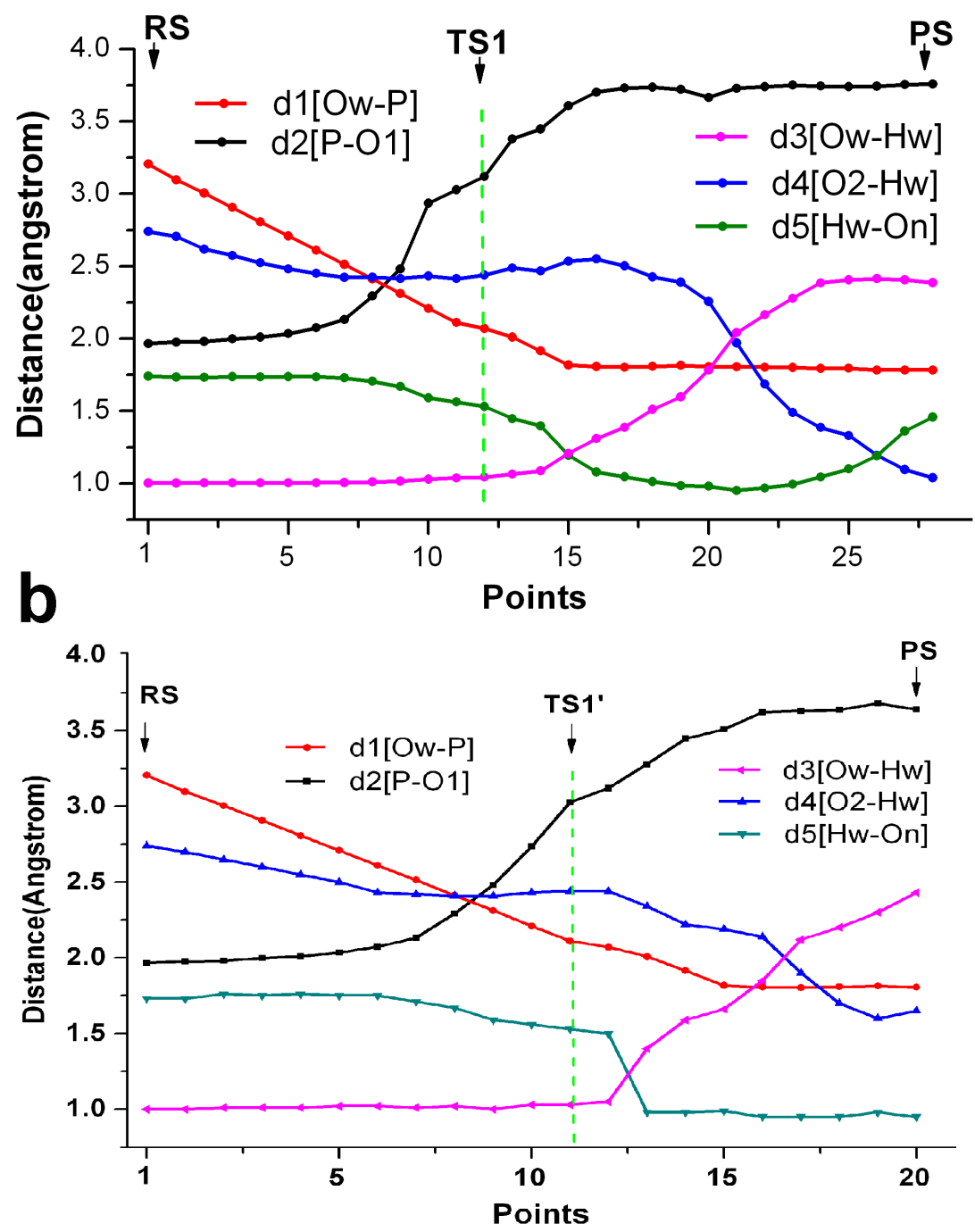
Figure 5. The details of the hydrogen-bond interactions specifically between the substrate and the protein ACP.

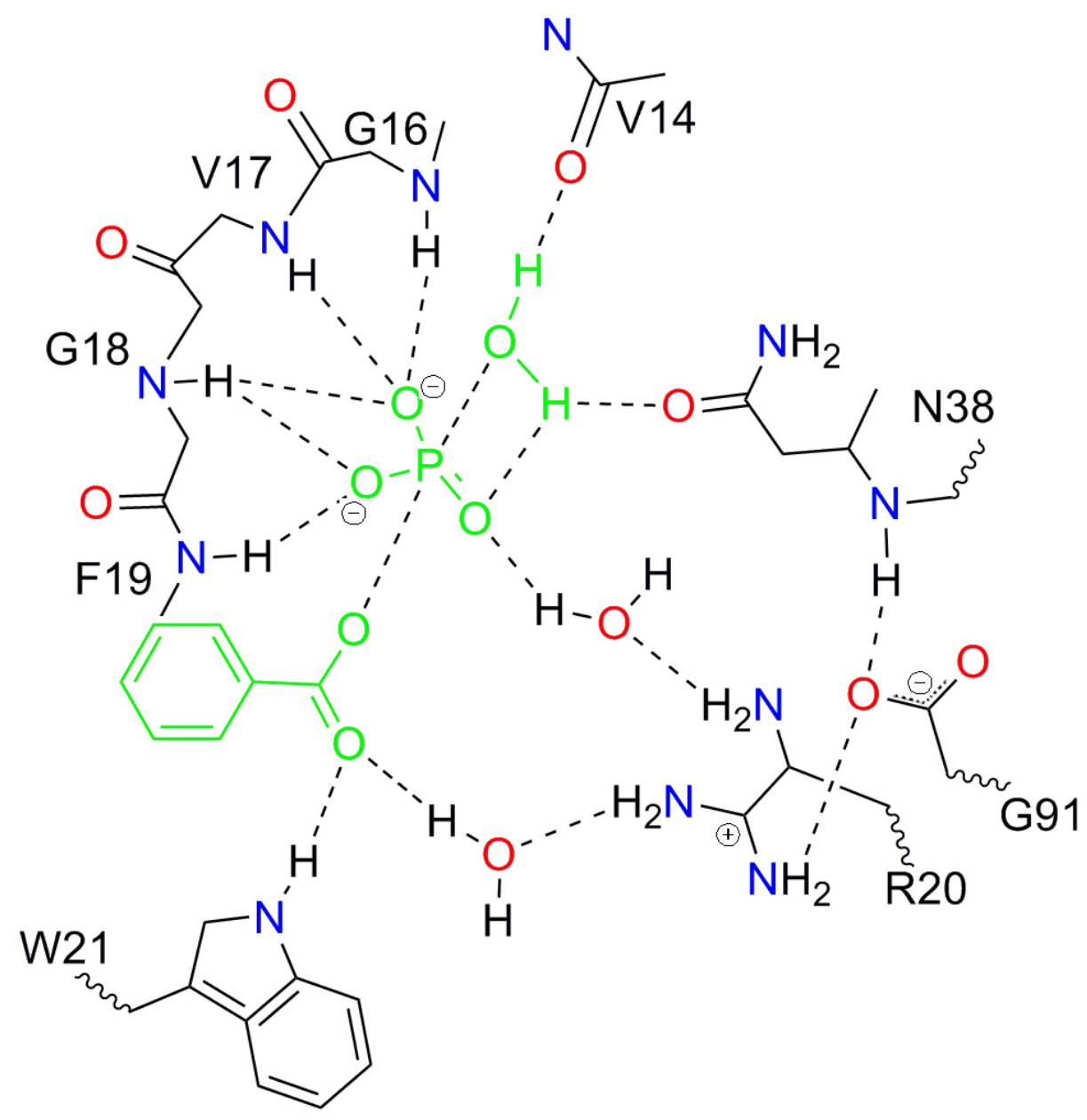


Figure S1. The complex model obtained by docking process.

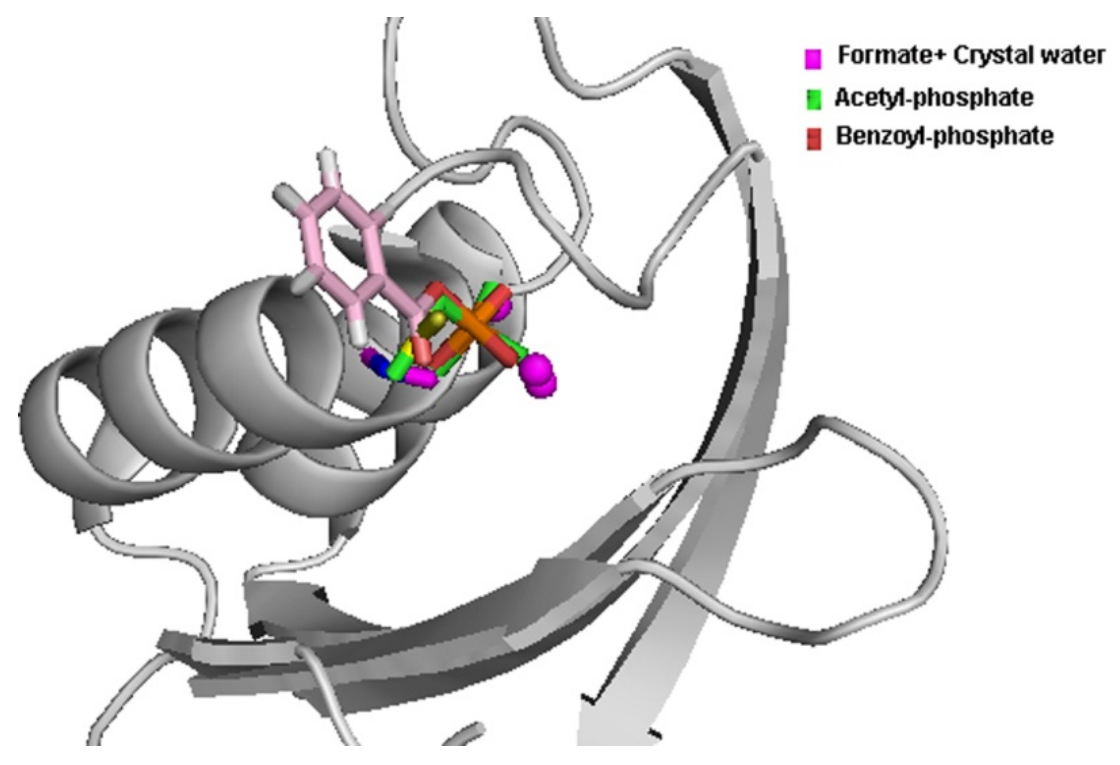


Figure S2. The RMSD of molecule dynamics of protein-substrate system. The panel shows the distribution of the RMSD for the trajectory in last $10 \mathrm{~ns}$.

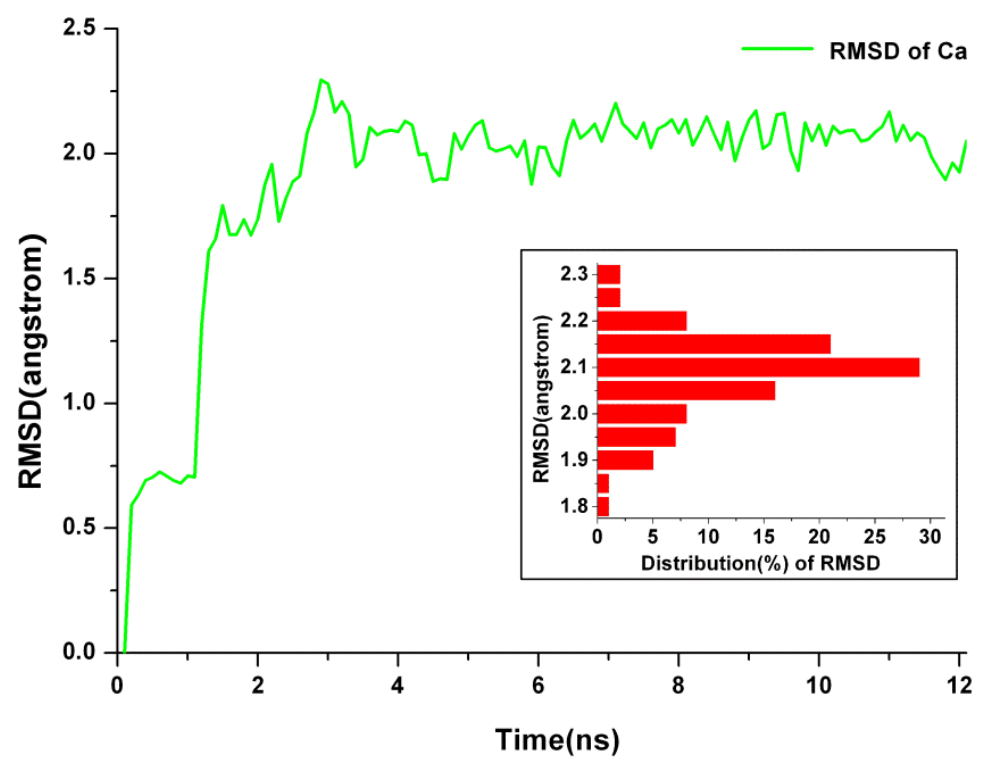

